\title{
LEBESGUE CONSTANTS FOR JACOBI EXPANSIONS ${ }^{1}$
}

\author{
DONALD I. CARTWRIGHT
}

\begin{abstract}
Sharp estimates are given for the Lebesgue constants $\left\|s_{n}\right\|_{p}=$ $\sup \left\{\left\|s_{n} f\right\|_{p}: f \in L_{*}^{p}\right.$, $\left.\|f\|_{p} \leqslant 1\right\}$ for $p$ outside the Pollard interval $\left(p_{0}^{\prime}, p_{0}\right)$, where $s_{n} f$ is the $n$th partial sum of the Jacobi expansion of a function $f$ which is in the $L^{p}$ space with respect to the weight $w(x)=(1-x)^{\alpha}(1+x)^{\beta}$ on $[-1,1]$.
\end{abstract}

1. Introduction and notation. For indices $\alpha, \beta \geqslant-\frac{1}{2}$, let $P_{n}^{(\alpha, \beta)}(x), n=0,1, \ldots$, be the Jacobi polynomials (see [9]) defined by the requirements that they are pairwise orthogonal relative to the weight $w(x)=w^{(\alpha, \beta)}(x)=(1-x)^{\alpha}(1+x)^{\beta}$ on $[-1,1]$, and satisfy $P_{n}^{(\alpha, \beta)}(1)=\left(\begin{array}{c}n+\alpha \\ n\end{array}\right)$ and $\operatorname{deg} P_{n}^{(\alpha, \beta)}=n$. Let $L_{w}^{p}, 1 \leqslant p \leqslant \infty$, denote the $L^{p}$ space with respect to the measure $d \mu=w(x) d x$ on $[-1,1]$, and let \|\|$_{p}$ denote the norm on $L_{w}^{p}$. The $n$th partial sum of the Jacobi expansion of a function $f \in L_{w}^{p}$ is

$$
\left(s_{n} f\right)(x)=\sum_{k=0}^{n} c_{k}(f) p_{k}(x),
$$

where $c_{k}(f)=\int_{-1}^{1} f(y) p_{k}(y) w(y) d y$ and $p_{k}(x)=\left(h_{k}^{(\alpha, \beta)}\right)^{-1 / 2} P_{k}^{(\alpha, \beta)}(x)$ and where $h_{k}^{(\alpha, \beta)}=\int_{-1}^{1}\left|P_{k}^{(\alpha, \beta)}(x)\right|^{2} w(x) d x$.

Pollard $[7,8]$ has shown that $s_{n} f \rightarrow f$ in the norm of $L_{w}^{p}$ provided that $p_{0}^{\prime}<p<p_{0}$, where $p_{0}=4(\gamma+1) /(2 \gamma+1), p_{0}^{\prime}$ is the index dual to $p_{0}$, and $\gamma=\max (\alpha, \beta)$. Furthermore, he showed that norm convergence does not hold for general $f \in L_{w}^{p}$ if $p \notin\left(p_{0}^{\prime}, p_{0}\right)$ (see [6] for the cases $\left.p=p_{0}, p_{0}^{\prime}\right)$. Of course the case $p_{0}=\infty$, i.e. $\alpha=\beta=-\frac{1}{2}$, for which the Jacobi expansion of $f$ is just the cosine expansion of $f(\cos \theta)$, had been well known.

In this paper we study the Lebesgue constants

$$
\left\|s_{n}\right\|_{p}=\sup \left\{\left\|s_{n} f\right\|_{p}: f \in L_{w}^{p} \text { and }\|f\|_{p} \leqslant 1\right\}
$$

for $p$ outside the Pollard interval $\left(p_{0}^{\prime}, p_{0}\right)$. Since $\left\|s_{n}\right\|_{p^{\prime}}=\left\|s_{n}\right\|_{p}$, we may assume that $p_{0} \leqslant p \leqslant \infty$.

THEOREM. Let $\alpha, \beta \geqslant-\frac{1}{2}, \gamma=\max (\alpha, \beta), p_{0}=4(\gamma+1) /(2 \gamma+1)$ and $\delta=$ $(2 \gamma+1) / 2-2(\gamma+1) / p$. Then there are numbers $A, B>0$, independent of $n$, such that

$$
A n^{\delta} \leqslant\left\|s_{n}\right\|_{p} \leqslant B n^{\delta} \text { for } p_{0}<p \leqslant \infty
$$

Received by the editors April 20, 1982.

1980 Mathematics Subject Classification. Primary 42C10.

Key' words and phrases. Jacobi polynomials, Lebesgue constants, compact symmetric spaces of rank one.

' Research partially supported by the C.N.R. 
and, when $n \geqslant 2$,

$$
A \log (n) \leqslant\left\|s_{n}\right\|_{p} \leqslant B \log (n) \text { for } p=p_{0} .
$$

For $p=\infty$ this result is known (see Light [5]). Furthermore, the left-hand inequality in (1) is a special case of a result of Dreseler and Soardi [3]. The left-hand inequality of (2) improves an estimate in [3].

After proving the theorem in the next section, we give an application of the result to harmonic analysis on compact symmetric spaces of rank one, in particular to Fourier analysis on $S U(2)$.

Throughout the paper, the letter $C$ denotes a positive constant which may be different from line to line.

\section{Proof of the theorem.}

Lemma. For real numbers $a, b, c$ such that $a c>-1, b c>-1$ and $c>1$, and for $0 \leqslant x<1$, we have

$$
\begin{aligned}
\int_{-1}^{1}\left|\frac{((1-y) /(1-x))^{a}((1+y) /(1+x))^{b}-1}{x-y}\right|^{c} d y \\
\leqslant \begin{cases}C /(1-x)^{\max (a c, c-1)} & \text { if } a c \neq c-1, \\
C|\log (1-x)| /(1-x)^{a c} & \text { if } a c=c-1 .\end{cases}
\end{aligned}
$$

When $-1<x \leqslant 0$, we obtain an estimate for the integral by replacing $x$ by $-x$ and $a$ by $b$ in the expression on the right.

Proof. We assume that $x \geqslant 0$, and (cf. [8]) consider the integral over four subintervals:

$$
\left(-1,-\left(\frac{1-x}{2}\right)\right),\left(-\left(\frac{1-x}{2}\right), x\right),\left(x, \frac{1+x}{2}\right) \text { and }\left(\frac{1+x}{2}, x\right) .
$$

In the first of these subintervals, $I_{1}$ say, $x-y \geqslant \frac{1}{2}$ holds, and so the integral over $I_{1}$ is $\leqslant C(1-x)^{-a c} \int_{I_{1}}(1+y)^{b c} d y+C \leqslant C(1-x)^{-a c}+C$ as $b c>-1$.

For $y$ in the second interval, $I_{2}$, we write

$$
\left|\left(\frac{1-y}{1-x}\right)^{a}\left(\frac{1+y}{1+x}\right)^{b}-1\right|^{c} \leqslant C\left(\frac{1+y}{1+x}\right)^{b c}\left|\left(\frac{1-y}{1-x}\right)^{a}-1\right|^{c}+C\left|\left(\frac{1+y}{1+x}\right)^{b}-1\right|^{c} \text {. }
$$

Now

$$
\left|\frac{((1+y) /(1+x))^{b}-1}{x-y}\right|^{c}=\left|\frac{((1+y) /(1+x))^{b}-1}{((1+y) /(1+x))-1}\right|^{c} \frac{1}{(1+x)^{c}}
$$

is bounded independently of $x \in[0,1]$ and $y \in I_{2}$, since $\frac{1}{2}<(1+y) /(1+x)<1$ for $y \in I_{2}$ and since $\left|z^{b}-1\right| \leqslant C|z-1| z^{\min (b .0)}$ for $0<z<1$. On the other hand,

$$
\left|\frac{((1-y) /(1-x))^{a}-1}{x-y}\right|^{c}=\left|\frac{((1-y) /(1-x))^{a}-1}{((1-y) /(1-x))-1}\right|^{c} \frac{1}{(1-x)^{c}}
$$


is bounded by $C(1-y)^{a c-c}(1-x)^{-a c}$ if $a>0$ and by $C(1-y)^{-c}$ if $a \leqslant 0$, since $(1-y) /(1-x)>1$ on $I_{2}$ and since $\left|z^{a}-1\right| \leqslant C|z-1| z^{\max (a, 0)-1}$ for $z>1$. The desired estimate follows.

An estimate $\int_{I_{3}} \leqslant C(1-x)^{-(c-1)}$ is obtained using the same technique used for estimating $\int_{I_{2}}$, together with the inequalities $1<(1+y) /(1+x) \leqslant 2$ and $\frac{1}{2}<$ $(1-y) /(1-x)<1$ valid on $I_{3}=(x,(1+x) / 2)$. The estimate $\int_{I_{4}} \leqslant C(1-x)^{-(c-1)}$ is easy to obtain, since $y-x \geqslant(1-x) / 2$ for $y \in I_{4}=((1+x) / 2,1)$, and since ac $>-1$.

We now turn to the proof of our theorem.

Let $p_{0} \leqslant p<\infty$, let $n>1$, and let $f \in L_{w}^{p}$ with $\|f\|_{p} \leqslant 1$. Our point of departure is the following formula for $\left(s_{n} f\right)(x)$ obtained by Pollard (formula (2.2) of [7]):

$$
\begin{aligned}
\left(s_{n} f\right)(x)= & \gamma_{n} p_{n+1}(x) \int_{-1}^{1} \frac{f(y) q_{n}(y) w(y)}{x-y} d y-\gamma_{n} q_{n}(x) \int_{-1}^{1} \frac{f(y) p_{n+1}(y) w(y)}{x-y} d y \\
& +\gamma_{n}^{\prime} p_{n+1}(x) \int_{-1}^{1} f(y) p_{n+1}(y) w(y) d y .
\end{aligned}
$$

Here $p_{n}(x)$ is as in $\S 1$, and $q_{n}(x)=\left(1-x^{2}\right)\left(h_{n}^{(\alpha+1, \beta+1)}\right)^{-1 / 2} P_{n}^{(\alpha+1, \beta+1)}(x)$, while $\gamma_{n}$ and $\gamma_{n}^{\prime}$, which are independent of $x$ and $p$, satisfy $\gamma_{n} \rightarrow \frac{1}{2}$ and $\gamma_{n}^{\prime} \rightarrow-\frac{1}{2}$. Let us write $\gamma_{n} F_{n}(x),-\gamma_{n} G_{n}(x)$ and $\gamma_{n}^{\prime} H_{n}(x)$ for the three terms of (3). The last of these is easy to dispose of. Indeed,

$$
\left\|\gamma_{n}^{\prime} H_{n}\right\|_{p} \leqslant C\left\|H_{n}\right\|_{p} \leqslant C\left\|p_{n+1}\right\|_{p}\left\|p_{n+1}\right\|_{p^{\prime}}\|f\|_{p} .
$$

But it is well known, and an easy consequence of $h_{n}^{(\alpha, \beta)} \sim n^{-1}$ and Theorem 7.32.2 of Szegö [9], that

$$
\left\|p_{n}\right\|_{p} \leqslant \begin{cases}C & \text { if } p<p_{0}, \\ C(\log n)^{1 / p_{0}} & \text { if } p=p_{0}, \\ C n^{\delta} & \text { if } p>p_{0}, \text { where } \delta=\frac{2 \gamma+1}{2}-\frac{2(\gamma+1)}{p} .\end{cases}
$$

(These estimates are in fact sharp (see [9, p. 391, Exercise 91]).) Thus $\left\|\gamma_{n}^{\prime} H_{n}\right\|_{p} \leqslant C n^{\delta}$ if $p>p_{0}$ and $\left\|\gamma_{n}^{\prime} H_{n}\right\|_{p} \leqslant C(\log n)^{1 / p_{0}}$ if $p=p_{0}$.

To estimate the first term of (3), we proceed initially as in [7]. We can write, using Theorem 7.32.2 of [9] again,

$$
p_{n+1}(x)=\frac{A_{n+1}(x)}{w(x)^{1 / 2}\left(1-x^{2}\right)^{1 / 4}} \text { and } q_{n}(y)=\frac{B_{n}(y)\left(1-y^{2}\right)^{1 / 4}}{w(y)^{1 / 2}}
$$

where $\left|A_{n+1}(x)\right| \leqslant C$ and $\left|B_{n}(y)\right| \leqslant C$. Thus

$$
\begin{aligned}
F_{n}(x)= & \frac{A_{n+1}(x)}{w(x)^{1 / p}} \int_{-1}^{1} w(y)^{1 / p} B_{n}(y) f(y) K(x, y) d y \\
& +\frac{A_{n+1}(x)}{w(x)^{1 / p}} \int_{-1}^{1} \frac{w(y)^{1 / p} B_{n}(y) f(y)}{x-y} d y,
\end{aligned}
$$


where

$$
K(x, y)=\frac{\left(\left(1-y^{2}\right) /\left(1-x^{2}\right)\right)^{1 / 4}(w(y) / w(x))^{1 / 2-1 / p}-1}{x-y} .
$$

It follows that

$$
\left|F_{n}(x)\right| \leqslant C \frac{\left|A_{n+1}(x)\right|}{w(x)^{1 / p}} \int_{-1}^{1} w(y)^{1 / p}|f(y)||K(x, y)| d y+E_{n}(X),
$$

where $\left\|E_{n}\right\|_{p} \leqslant C$. Applying the lemma with $a=\frac{1}{4}+\alpha\left(\frac{1}{2}-\frac{1}{p}\right), b=\frac{1}{4}+\beta\left(\frac{1}{2}-\frac{1}{p}\right)$ and $c=p^{\prime}$ (noting that $a, b \geqslant 1 / 2 p>0$ so that $a c, b c>-1$ ), we see that the integral in (6) is, for $x \geqslant 0$, at most

$$
\left(\int_{-1}^{1}|K(x, y)|^{p^{\prime}} d y\right)^{1 / p^{\prime}} \leqslant \begin{cases}C /(1-x)^{\max (a, 1 / p)} & \text { if } a \neq \frac{1}{p} \\ C|\log (1-x)|^{1 / p^{\prime}} /(1-x)^{1 / p} & \text { if } a=\frac{1}{p} .\end{cases}
$$

Using $\left|A_{n+1}(x)\right| \leqslant C$ for $x \in\left[0,1-n^{-2}\right]$ and

$$
\left|A_{n+1}(x)\right|=\left|p_{n+1}(x)\right| w(x)^{1 / 2}\left(1-x^{2}\right)^{1 / 4} \leqslant C n^{\alpha+1 / 2}(1-x)^{(2 \alpha+1) / 4}
$$

for $x \in\left[1-n^{-2}, 1\right]$, we therefore have (for $a \neq \frac{1}{p}$ ),

$$
\begin{aligned}
\int_{0}^{1} \mid & \left.F_{n}(x)\right|^{p} w(x) d x \leqslant C \int_{0}^{1-n^{-2}} \frac{d x}{(1-x)^{\max (a p, 1)}} \\
& +C n^{(\alpha+1 / 2) p} \int_{1-n^{-2}}^{1}(1-x)^{((2 \alpha+1) / 4) p-\max (a p, 1)} d x+C \\
\leqslant & C \max \left\{\log n, n^{(\alpha+1 / 2) p-2(\alpha+1)}\right\} \\
\leqslant & C \max \left\{\log n, n^{p \delta}\right\} .
\end{aligned}
$$

If $a=\frac{1}{p}$, i.e. $p=4(\alpha+1) /(2 \alpha+1)$, however, we have

$$
\begin{aligned}
\int_{0}^{1}\left|F_{n}(x)\right|^{p} w(x) d x \leqslant & C \int_{0}^{1-n^{-2}} \frac{|\log (1-x)|^{p / p^{\prime}}}{1-x} d x \\
& +C n^{(\alpha+1 / 2) p} \int_{1-n^{-2}}^{1}(1-x)^{\alpha}|\log (1-x)|^{p / p^{\prime}} d x+C .
\end{aligned}
$$

The first of these terms on the right is $\leqslant C(\log n)^{p}$ since $|\log (1-x)| \leqslant 2 \log n$ for $x \in\left[0,1-n^{-2}\right]$. With only slightly more effort we see that the second term is $\leqslant C(\log n)^{p / p^{\prime}} n^{(\alpha+1 / 2) p-2(\alpha+1)}=C(\log n)^{p-1}$. The integral $\int_{-1}^{0}\left|F_{n}(x)\right|^{p} d x$ is estimated in the same way, so we obtain that the norm of the first term $\gamma_{n} F_{n}$ of (3) is $\leqslant C n^{\delta}$ for $p>p_{0}$, and $\leqslant C \log n$ for $p=p_{0}$.

The second term $-\gamma_{n} G_{n}$ of (3) is treated in the same way as the first term. The lemma is applied again with $c=p^{\prime}, a=-\frac{1}{4}+\alpha\left(\frac{1}{2}-\frac{1}{p}\right)$ and $b=-\frac{1}{4}+\beta\left(\frac{1}{2}-\frac{1}{p}\right)$, so that $a c, b c \geqslant-\frac{1}{2}>-1$. We find that

$$
\left\|G_{n}\right\|_{p} \leqslant \begin{cases}C \max \left\{(\log n)^{1 / p}, n^{\delta-1}\right\} & \text { if } a, b \neq \frac{1}{p}, \\ C \log n & \text { if } a \text { or } b=\frac{1}{p} .\end{cases}
$$


The right-hand inequalities in (1) and (2) are thus proved. For the left-hand inequality in (1), we refer to [3]. Let us now consider the case $p=p_{0}$. In this case $\left\|G_{n}\right\|_{p} \leqslant C(\log n)^{1 / p}$, since $-\frac{1}{4}+\alpha\left(\frac{1}{2}-\frac{1}{p}\right)$ and $-\frac{1}{4}+\beta\left(\frac{1}{2}-\frac{1}{p}\right)$ are $\leqslant-\frac{1}{4}+$ $\gamma\left(\frac{1}{2}-\frac{1}{p}\right)=\frac{1}{p}-\frac{1}{2}<\frac{1}{p}$.

Let us now suppose that $\beta \leqslant \alpha$ and let us estimate $\left\|s_{n} f\right\|_{p}^{p} \geqslant \int_{0}^{1}\left|\left(s_{n} f\right)(x)\right|^{p} d x$ from below. (If $\beta>\alpha$, we estimate $\int_{-1}^{0}\left|\left(s_{n} f\right)(x)\right|^{p} d x$ instead.) If we examine the proof of the lemma and of the estimate $\int_{0}^{1}\left|F_{n}(x)\right|^{p} d x \leqslant C(\log n)^{p}$, we see from (5) that

$$
\begin{aligned}
\left(s_{n} f\right)(x)= & \gamma_{n} \frac{A_{n+1}(x)}{w(x)^{1 / p}} \int_{0}^{x} w(y)^{1 / p} B_{n}(y) f(y)\left(\frac{1+y}{1+x}\right)^{h} \\
& \cdot\left(\frac{((1-y) /(1-x))^{a}-1}{x-y}\right) d y+\tilde{E}_{n}(x)
\end{aligned}
$$

where $\int_{0}^{1}\left|\tilde{E}_{n}(x)\right|^{p} d x \leqslant C \log n, a=\frac{1}{4}+\alpha\left(\frac{1}{2}-\frac{1}{p}\right)=\frac{1}{p}$, and $b=\frac{1}{4}+\beta\left(\frac{1}{2}-\frac{1}{p}\right)$. Now for $0<y<x$ we have

$$
\begin{aligned}
\frac{((1-y) /(1-x))^{1 / p}-1}{x-y} & =\frac{((1-y) /(1-x))^{1 / p}-1}{(1-y) /(1-x)-1} \frac{1}{1-x} \\
& \geqslant \frac{1}{p}\left(\frac{1-y}{1-x}\right)^{1 / p-1} \frac{1}{1-x} .
\end{aligned}
$$

If we take $n$ large enough that $\gamma_{n} \geqslant \frac{1}{4}$ and set

$$
f(y)=\operatorname{sign}\left(B_{n}(y)\right)\{2 \log (n)(1-y) w(y)\}^{-1 / p}
$$

for $0 \leqslant y \leqslant 1-n^{-2}$ and $f(y)=0$ otherwise, we see that $\|f\|_{p}=1$ and that, for $0 \leqslant x \leqslant 1-n^{-2}$,

$$
\begin{aligned}
& \left|\left(s_{n} f\right)(x)\right| \geqslant C \frac{\left|A_{n+1}(x)\right|}{\{\log (n) w(x)(1-x)\}^{1 / p}} \int_{0}^{x} \frac{\left|B_{n}(y)\right|}{1-y} d y-\left|\tilde{E}_{n}(x)\right| \\
& \quad \geqslant \frac{C(1-x)^{\alpha / p}\left|p_{n+1}(x)\right|}{\{\log (n) w(x)\}^{1 / p}} \int_{0}^{x}(1-y)^{(\alpha+1) / 2-3 / 4}\left|r_{n}(y)\right| d y-\left|\tilde{E}_{n}(x)\right|,
\end{aligned}
$$

where $r_{n}(y)=\left(h_{n}^{(\alpha+1, \beta+1)}\right)^{-1 / 2} P_{n}^{(\alpha+1, \beta+1)}(y)$. It is a fairly routine application of the asymptotic formula 8.21 .18 of [9] to see that

$$
\int_{0}^{x}(1-y)^{(\alpha+1) / 2-3 / 4}\left|r_{n}(y)\right| d y \geqslant C|\log (1-x)|
$$

for $x_{0} \leqslant x \leqslant 1-d n^{-2}$ and $n \geqslant n_{0}$, where $n_{0}, x_{0}, d$ and $C$ are suitable positive constants, with $x_{0}<1$. Another application of the asymptotic formula shows from 
(7) that

$$
\begin{aligned}
\int_{x_{0}}^{1-d n^{-2}} \mid & \left.\left(s_{n} f\right)(x)\right|^{p} d x \\
& \geqslant \frac{C}{\log n} \int_{x_{0}}^{1-d n^{-2}}\left|p_{n+1}(x)\right|^{p}(1-x)^{\alpha}|\log (1-x)|^{p} d x-C \log n \\
& \geqslant C(\log n)^{p} .
\end{aligned}
$$

This completes the proof of the theorem.

3. Application to compact symmetric spaces of rank one. We mention briefly an application of our theorem to harmonic analysis on compact symmetric spaces $M$ of rank one. It is well known (see, e.g., [1]) that for some $\alpha \geqslant \beta \geqslant-\frac{1}{2}$ there is, for each $1 \leqslant p \leqslant \infty$, an isometry between $L_{z}^{p}(M, \mu)$ and $L_{w}^{p}$, where $w=w^{(\alpha, \beta)}$. Here $\mu$ is the normalized measure on $M$ which is invariant with respect to the group of isometries of $M$, and $L_{z}^{p}(M, \mu)$ denotes the subspace of $L^{p}(M, \mu)$ consisting of functions $f$ which are zonal with respect to a fixed point $\xi_{0} \in M$ (i.e. for which $f(\xi)$ depends only on the geodesic distance from $\xi$ to $\xi_{0}$ ). The Jacobi expansion of a function $\tilde{f}$ in $L_{n}^{p}$. corresponds to the zonal harmonic expansion $\sum_{n=0}^{\infty} f * Z^{n}$ of the corresponding zonal function $f$. The proof of Theorem B of [2] carries over to the $L^{p}$ case, where $p_{0}<p<\infty$, with only slight modifications and yields the following result, in which $E^{(s)}$ and $\omega_{2}\left(t, f^{(s)}\right)$ are the $L^{p}$ versions of, respectively, the space of $s$-times differentiable functions $\left(E=L^{p}(M, \mu)\right)$ and the modulus of continuity defined in $[2]$.

COROllary. Let $M$ be a compact symmetric space of rank one and dimension $d \geqslant 2$. Let $\alpha=(d-2) / 2$ and $\infty>p>p_{0}=4(\alpha+1) /(2 \alpha+1)$ and $\delta=(2 \alpha+1) / 2-$ $2(\alpha+1) / p$.

(a) If $f \in E^{(s)}$ is zonal and $\omega_{2}\left(t, f^{(s)}\right)=o\left(t^{\delta-s}\right)$ as $t \rightarrow 0$ for some integer $s \geqslant 0$, then $\sum_{n=0}^{N} f * Z^{n} \rightarrow f$ in the norm of $L_{z}^{p}(M, \mu)$.

(b) On the other hand, there exists a zonal function $f \in E^{(s)}$, where $s$ is the largest integer $<\delta$, such that $\omega_{2}\left(t, f^{(s)}\right)=O\left(t^{\delta-s}\right)$ as $t \rightarrow 0$ but such that $\sum_{n=0}^{N} f * Z^{n}$ does not converge to $f$ in the norm of $L_{z}^{p}(M, \mu)$.

The case $M=S U(2) \cong S^{3}$, the 3-sphere, is of particular interest. Here $\alpha=\beta=\frac{1}{2}$, so that $p_{0}=3$ and $\delta=1-\frac{3}{p}$. A function $f$ on $S U(2)$ which is zonal with respect to the identity is just a central function, and its zonal harmonic expansion is its Fourier expansion. The estimates (1) and (2) are thus estimates for the Lebesgue constants

$$
\sup \left\{\left\|\left(\sum_{k=0}^{n}(k+1) \chi_{k}\right) * f\right\|_{p}: f \in L^{p}(S U(2)), f \text { central, }\|f\|_{p} \leqslant 1\right\} \text {, }
$$

where $\chi_{k}$ is the character of the irreducible representation of $S U(2)$ of degree $k+1$. Our estimates here for the Lebesgue constants of $S U(2)$ sharpen those obtained by Giulini, Soardi and Travaglini [4] for general compact connected Lie groups. 


\section{REFERENCES}

1. R. Askey and N. H. Bingham, Gaussian processes on compact symmetric spaces, Z. Wahrsch. Verw. Gebiete 37 (1976), 127-143.

2. D. I. Cartwright and P. M. Soardi, Best conditions for the norm convergence of Fourier series (preprint).

3. B. Dreseler and P. M. Soardi, A Cohen type inequality for Jacobi expansions and divergence of Fourier series on compact symmetric spaces, J. Approx. Theory 35 (1982), 214-221.

4. S. Giulini, P. M. Soardi and G. Travaglini, Norms of characters and Fourier series on compact Lie groups, J. Funct. Anal. 46 (1982), 88-101.

5. W. A. Light, Jacobi projections, Approximation Theory and Applications (Zvi Ziegler, ed.), Academic Press, New York, 1981.

6. J. Newman and W. Rudin, Mean convergence of orthogonal series, Proc. Amer. Math. Soc. 3 (1952), 219-222.

7. H. Pollard, The mean convergence of orthogonal series. II, Trans. Amer. Math. Soc. 63 (1948), 355-367.

8. The mean convergence of orthogonal series. III, Duke Math. J. 16 (1949), 189-191.

9. G. Szegö, Orthogonal polynomials, 4th ed., Amer. Math. Soc. Colloq. Publ., vol. 23, Amer. Math. Soc., Providence, R. I., 1975.

Department of Pure Mathematics, The University of Sydney, Sydney, N.S.W. 2006, Australia 